\title{
A SUPERCONDUCTING MICROWAVE ENGINE
}

$$
\text { G. J. Dick }{ }^{*}
$$

\section{ABS TRAC T}

In this paper a new technique of electromechanical energy conversion is proposed. This technique would make use of the high Q's attainable in superconducting resonators to achieve direct mechanical to microwave energy conversion with a net efficiency greater than $90 \%$, a value which is far higher than that obtained by conventional techniques. In addition, if surface magnetic and electric field levels are limited by the critical fields obtained in fixed resonators, power densities would be achieved which are large enough to make such a machine a practical means both for generating microwave energy and for re-converting the microwave again to mechanical energy for power transmission purposes.

\section{INTRODUCTION}

In this paper, a new technique of electromechanical energy conversion will be discussed involving the "compression" of an electromagnetic field. It is well known that magnetic fields cause forces and indeed the expression for the pressure produced by a magnetic field $B$ is just $\frac{1}{2} B^{2}$, or a pressure of about 50 pounds per square $\mu_{0}$ inch (PSI) for a static $10 \mathrm{kG}$ field. However, oscillating magnetic fields also cause forces of the same type--in an rf resonator containing a $1 \mathrm{kG}$ oscillating field the radiation pressure on the walls is about $1 / 2$ PSI. For the sake of illustration, suppose this radiation were contained in an ideal resonator with perfectly flexible walls. In this case, a counterbalancing force on the walls in the same amount would be required to preserve equilibrium. However, in this ideal resonator, if the external force were increased slightly, the volume of the resonator would contract and work would be done on the electromagnetic fields contained inside. This work would show up as an increase in the stored electromagnetic energy.

The particular form which is taken by the energy in the resonator is described by the Boltzmann-Ehrenfest Theorem. ${ }^{1}$ This theorem states that under conditions of adiabatic (slow) deformation, the number of photons contained in a lossless resonator remains constant. That is, the energy is given by $\mathrm{E}=\mathrm{N}$ w where $\mathrm{N}$ is the number of photons in the cavity and $\omega$ is the instantaneous eigenfrequency. Thus, if we could compress a cavity by a factor of 10 in a time much shorter than that required for the electromagnetic energy to decay, the frequency and energy content of the cavity would both increase ten times. If such a procedure could be repeated at a high rate, a small amount of power at $10 \mathrm{w}$ microwave frequency could be converted into a larger amount of power at a higher frequency. That is, an engine could be built to "generate" microwave power from lower frequency energy, or conversely, to convert microwave power into a combination of mechanical work and lower frequency energy.

To build such an engine, the eigenfrequency must be changed in a time short compared to the electromagnetic ringing time. This is not possible using nor-

Manuscript received September 30, 1974

* Work supported by the office of Naval Research, Contract No. N00014-70-C-0133 and the National Science Foundation Grant No. GP-37166. R.A.I., So. Laguna, CA. 92677 and California Institute of Technology, Low Temperature Physics 63-37, Pasadena, California 91125. mal resonators, which have ringing times of microseconds. However, it may be feasible in a superconducting resonator where ringing times of hundreds of milliseconds or even seconds can be achieved.

In order to achieve a rapid deformation of the superconducting resonator, it would need to be built in two parts, one of which would be rotating very rapidly past the other. If the technical problems of cooling the rotor in such a machine could be solved and if the strength of materials appropriate to the construction of this rotor can be made large enough, it would be possible to construct a resonator with a very rapidy changing eigenfrequency.

In the following section, we will present a preliminary analysis of the efficiency which could be obtained under these conditions for energy conversion, and make a rough guess at the power levels expected.

\section{ANALYSIS}

A schematic diagram of the microwave engine is shown in Fig. 1. When the cavity reaches it low frequency position, the first switch closes and the cavity is excited with electromagnetic energy $E_{\text {, at }}$ a frequency $\omega_{1}$. This switch is then opened while the eigenfrequency of $\omega_{2}$ he cavity changes to a new value $\omega_{2}$. Then energy $\mathrm{E}_{2}=\frac{\omega_{2}}{\omega_{1}} \mathrm{E}_{1}$ is coupled out of the cavity by closby closing $\omega_{1}$ the second switch. During the time that the cavity is excited, some fraction of the energy will be absorbed by losses in the cavity walls. Furthermore, the energy density in the cavity will be limited by the maxinum magnetic and electric fields which can be sustained at the superconducting surface. We evaluate the limits these considerations place on the efficiency and power density as follows.

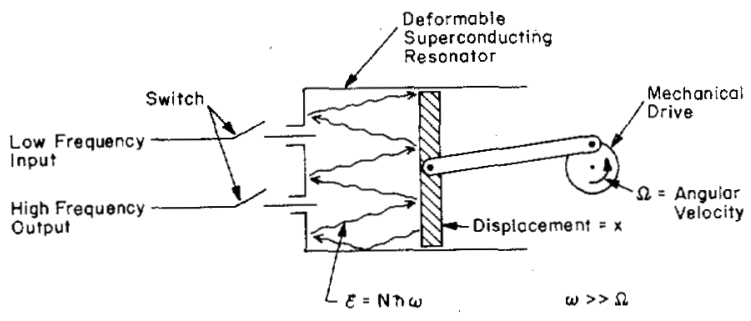

Fig. 1. Schematic diagram of Microwave Engine using a variable frequency superconducting resonator as the frequency conversion element. Under adiabatic conditions $(\omega>\Omega)$ the number of photons $N$ in a lossless cavity remains constant.

The fractional loss of microwave energy at helium temperature is given by $T / T$ where $T$ is the time spent by the energy in the cavity, and $T$ is the decay time for the resonator. However, it takes approximately 1000 watts of power for refrigeration for each watt dissipated at helium temperature. Thus, the net efficiency $€$ is given

$$
\varepsilon=1-10^{3} \mathrm{~T} / \tau
$$


The decay time for energy in a resonator with resistive losses can be written in the form

$$
\tau=\frac{\mu_{0}}{2} \frac{L_{1}}{R_{s}}
$$

where $\mathrm{L}_{7}$ is a characteristic size of the resonator (the volume to surface ratio) and $R_{\text {is }}$ is the surface resistance. The time which the microwaves must spend in the cavity, $T$ is approximately equal to half the time for a mechanical cycle, and is given approximately by

$$
\mathrm{T}=\frac{\mathrm{L}_{2}}{2 \mathrm{v}}
$$

where $L_{2}$ is again a characteristic size of the resonator and $v$ is the velocity with which one part of the resonator moves past another. If we set $\alpha=L_{2} / L_{1}$, we obtain a value for the efficiency which is Independent of size,

$$
\varepsilon=1-\frac{10^{3} \times R_{s}}{\mu_{0} v} \alpha
$$

In a similar manner, the power is given by the energy content $\mathrm{E}$, in the in the resonator divided by the time per cycle, or

$$
\mathrm{P}=\frac{\mathrm{E}}{2 T}=\frac{\left(\mathrm{B}_{\mathrm{c}}^{2} / 2 \mu_{\mathrm{o}}\right) \times \mathrm{V}}{\mathrm{L}_{2} / v}
$$

where $V$ is the volume of the resonator and $B$ is the limiting magnetic field at the superconducting surface. Using the same definition of $\alpha$ as before, we obtain,

$$
P=\frac{B_{c}{ }^{2}}{2 \mu_{0} \alpha} \times A
$$

where $\mathrm{A}$ is the resonator area.

\section{DISCUSSION}

In order to evaluate the potential efficiencies and power levels possible with this technique, we must make guesses of the surface properties, bulk strength, and electromagnetic field configurations which can be combined in a low temperature machine. We can estimate with some confidence the electrical characteristics which can be achieved at superconducting surfaces in the absence of the stresses which would be introduced in a rapidly spinning superconducting rotor. Surface resistances of $10^{-\delta} \Omega$ have been achieved at fields approach1ng $1000 \mathrm{~g}$ at a number of frequencles and low level losses of $10^{-9} \Omega$ have been observed.

On the other hand, the technology of moving one part rapidly past another has been thoroughly developed in the design of low pressure steam turbines. Rotor speeds of $300 \mathrm{M} / \mathrm{sec}$ are routine, even taking into account a $25 \%$ overspeed safety factor and the weakening effects of the high temperatures involved. "We estimate that this same speed can be attained in a superconducting machine. The properties of the resonator itself determine the parameter $\alpha$ and are a little harder to guess. The specific form of the resonator will be heavily dependent on mechanical considerations. Based on some simple cylindrical models, we estimate $\alpha \approx 4$.

Using these parameters, we estimate an overall efficiency from Eq. (4) between $90 \%$ and $99 \%$ depending on which value of surface resistance is used. If an average field of $700 \mathrm{~g}$ could be sustained in the resonator, the power density given by Eq. (6) would be approximately $1 \times 10^{5}$ watts per square meter of resonator surface area.

These efficiencies are far higher than those presently achievable for either microwave generation or rectification. In fact, the highest overall efficiency presently demonstrated by a microwave power link is approximately $40 \% .3$ The availability of a means to generate and rectify microwaves at efficiencies comparable to these attalned by other power techniques would make microwave power transmission a realistic posstbility.

\section{CONCLUSION}

We have proposed a possible use of superconducting resonators to convert microwave power at a low frequency to a larger amount of power at a high frequency, the difference in energy being provided by mechanical work done by the cavity walls on the fields. Although the development of such an application would involve considerable technological accomplishments, the present state of the art of rf superconductivity could make possible moderate power levels and high efficiencies for the generation and conversion to mechanical work of microwave energy. The capability for such energy conversion could open important new applications for microwave power.

\section{ACKNOWLEDGEMENTS}

The author would like to express his gratitude to J. E. Mercereau and $K$. W. Shepard for their support and contributions to this work.

\section{REFERENCES}

1. See Electromagnetic Waveguides and Resonators by F. E. Borgnis and C. H. Papas in Handbuck der Physik V.. 16, (Springer-Verlag, Ber1in) 1958.

2. Kent's Mechanical Engineer's Handbook, 12th edition. V.II, J. Kenneth Salisbury, Ed. (W1ley, New York). 1967.

3. Feasibility Study of a Satellite Solar Power Station. NASA Contractor Report, $\frac{\text { NASA CR } 2357 .}{23}$ 